Figure 1:

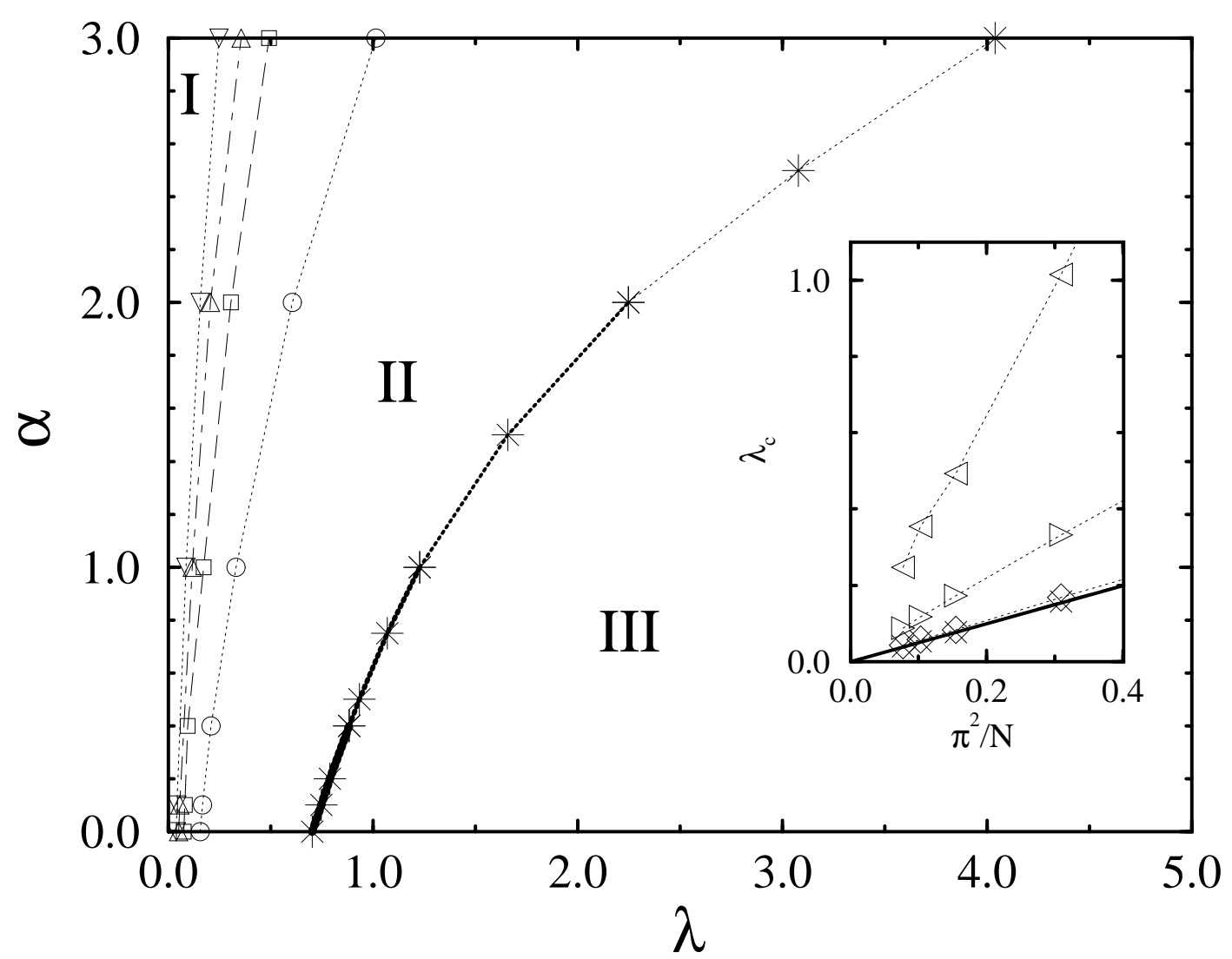


Figure 2:
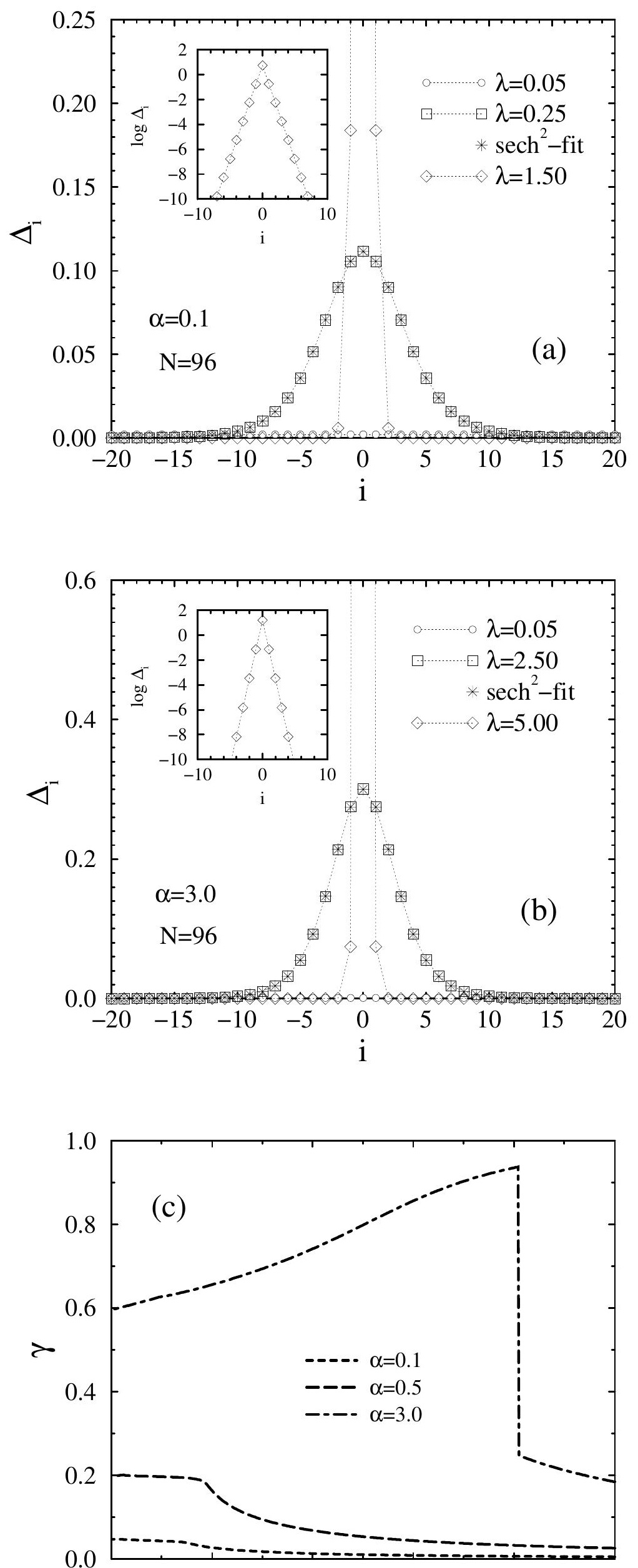
Figure 3:
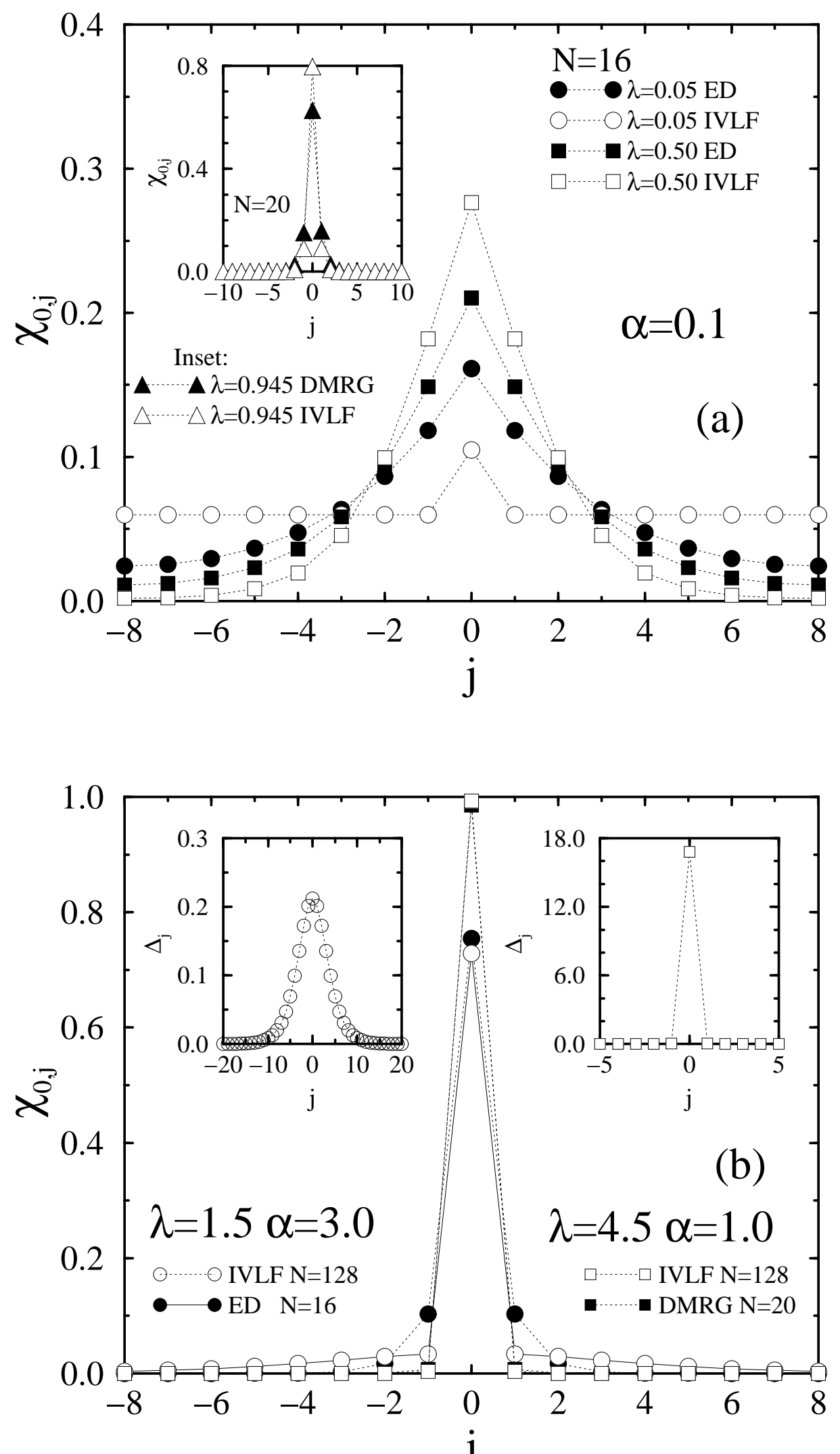
Figure 4:
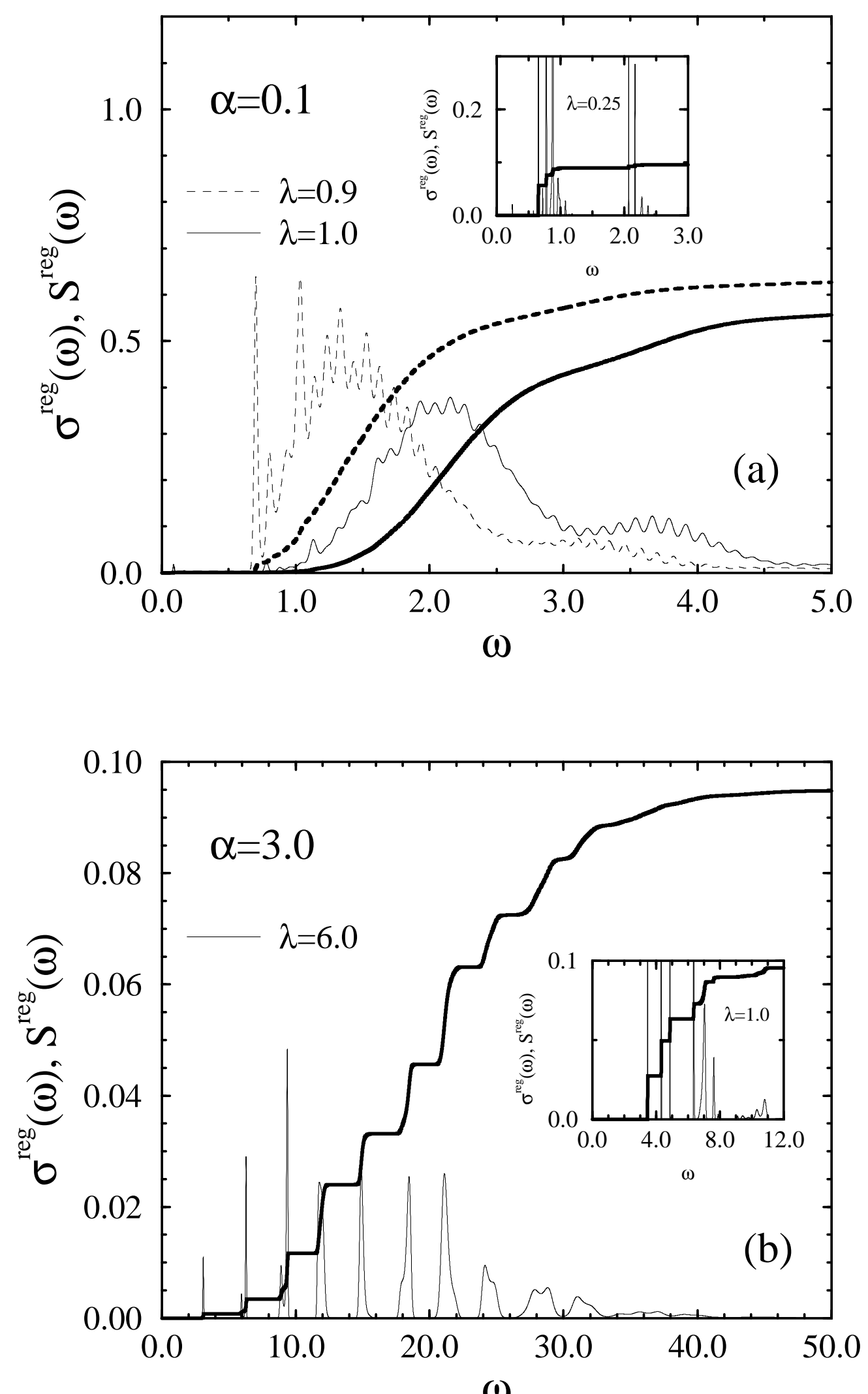
Figure 5:
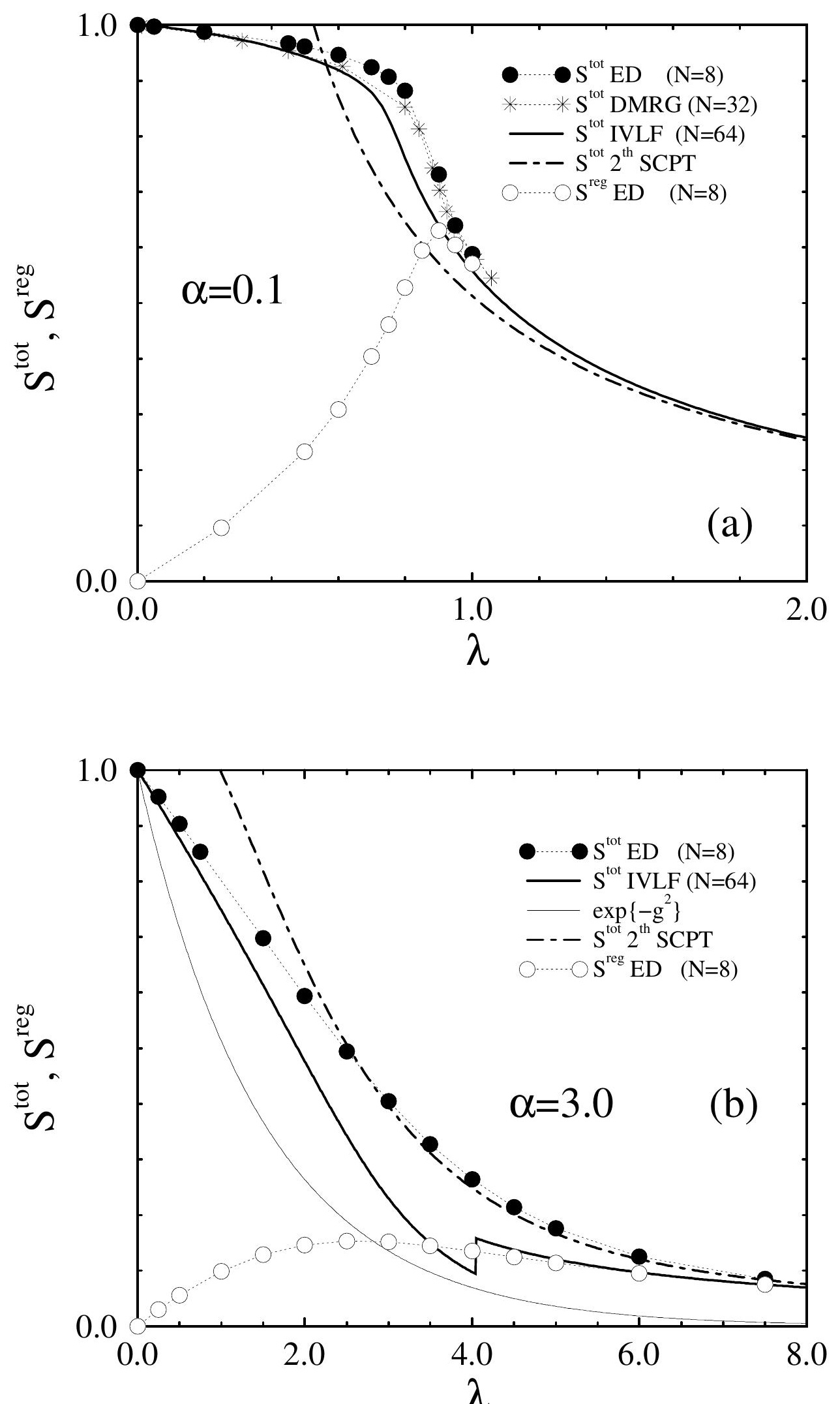
Figure 6:

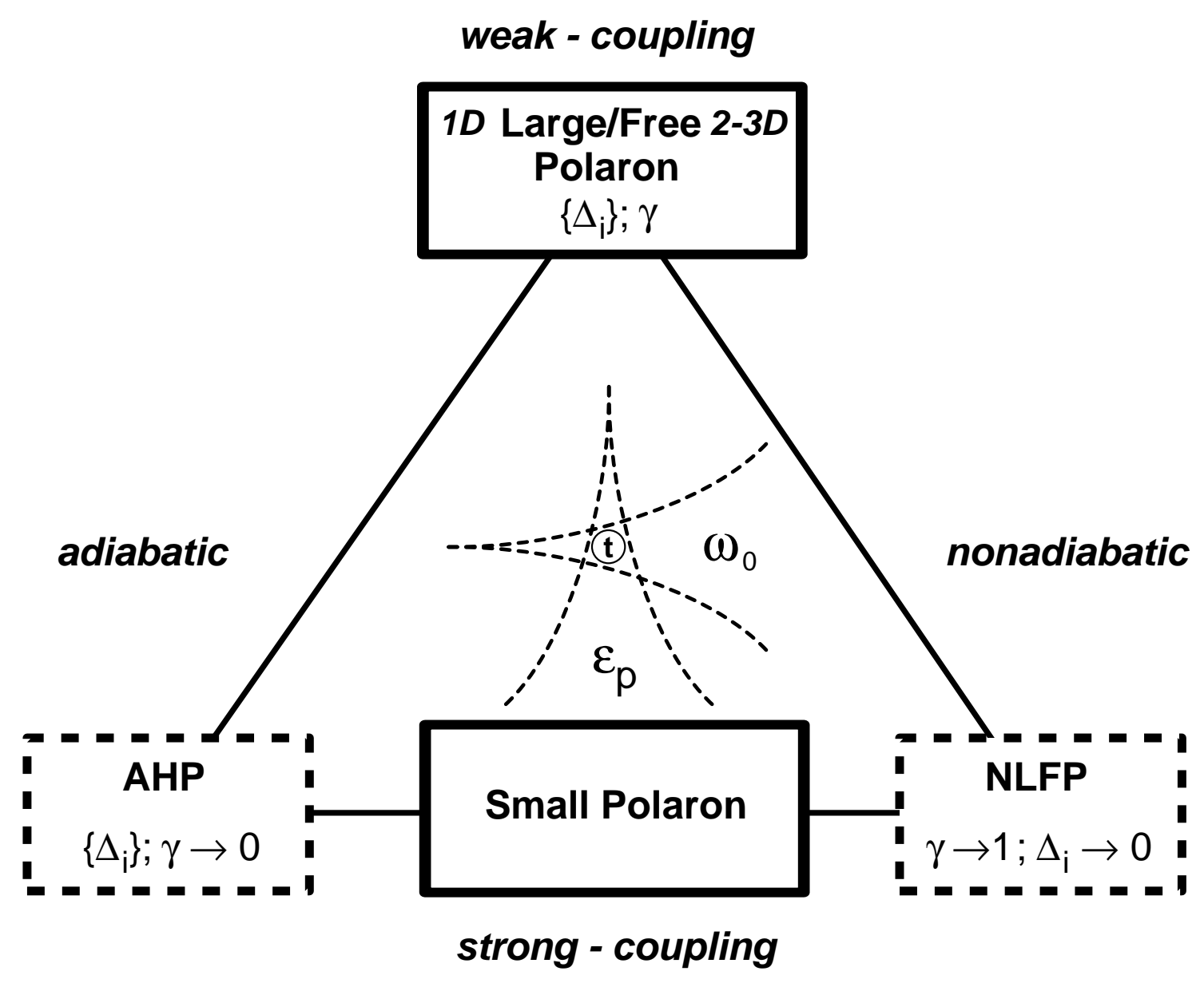




\title{
On the self-trapping problem of electrons or excitons in one dimension
}

\author{
G. Wellein and H. Fehske \\ Physikalisches Institut, Universität Bayreuth, D-95440 Bayreuth, Germany
}

(Bayreuth, 19 December 1997)

\begin{abstract}
We present a detailed numerical study of the one-dimensional Holstein model with a view to understanding the self-trapping process of electrons or excitons in crystals with short-range particle-lattice interactions. Applying a very efficient variational Lanczos method, we are able to analyze the groundstate properties of the system in the weak- and strong-coupling, adiabatic and non-adiabatic regimes on lattices large enough to eliminate finite-size effects. In particular, we obtain the complete phase diagram and comment on the existence of a critical length for self-trapping in spatially restricted onedimensional systems. In order to characterize large and small polaron states we calculate self-consistently the lattice distortions and the particle-phonon correlation functions. In the strong-coupling case, two distinct types of small polaron states are shown to be possible according to the relative importance of static displacement field and dynamic polaron effects. Special emphasis is on the intermediate coupling regime, which we also study by means of direct diagonalization preserving the full dynamics and quantum nature of phonons. The crossover from large to small polarons shows up in a strong decrease of the kinetic energy accompanied by a substantial change in the optical absorption spectra. We show that our numerical results in all important limiting cases reveal an excellent agreement with both analytical perturbation theory predictions and very recent density matrix renormalization group data.
\end{abstract}




\section{INTRODUCTION}

Electrons, holes or excitons delocalized in a perfect rigid lattice can be "trapped" within a potential well produced by displacements of atoms from their carrier-free equilibrium positions, provided the particle-lattice interaction is sufficiently strong [1] [4]. Trapping of a carrier in this manner is more advantageous energetically as compared to wide-band Bloch states, if the lowering of the carrier's energy due to its binding exceeds the strain energy required to produce the trap. Since the potential that binds the carrier depends on the carrier's state itself, i.e., the local distortion of the lattice is self-induced by the particle, this process is called "self-trapping" or "auto-localization". Obviously, self-trapping (ST) is an highly non-linear phenomenon. A self-trapped state is referred to as "large" if it extends over multiple lattice sites. Alternatively, if the quasiparticle is practically confined to a single site the ST state is designated as "small". Nonetheless, ST does not imply a breaking of translational invariance. ST eigenstates in a crystal are Bloch-like. Thus coherent transport of ST particles can, in principle, occur but the width of the corresponding band is extremely small (cf. the discussion in [5]).

Introducing the concept of polarons into physics, the possibility of electron immobilization or ST was pointed out by Landau as early as 1933 [6]. Shortly after the ST of excitons was also suggested and studied theoretically [7],8]. ST polarons and excitons can be found in a wide variety of alkali metal halides, alkaline earth halides, II-IV and group IV semiconductors, condensed rare gases, organic molecular crystals, electrochromics, and other systems [3,9]. With the observation of polaronic effects in new materials exhibiting exceptional properties such as the high- $T_{c}$ cuprates [10 or the colossal magnetoresistance manganates [11], research on polarons has attracted renewed attention.

Although the problems of exciton and electron ST have much in common there are fundamental differences. Most notably excitons are short-living non-equilibrium quasiparticles being immediately after the optical excitation in the free state and can reach the ST state only by tunneling through the potential barrier at low temperatures. Moreover, if 
the electron and hole, forming the exciton, have very different effective masses, the internal coordinates of this large-radius (Wannier-Mott) exciton will be of importance.

It is clear that the microscopic structure of the ST state is very diverse in various groups of materials. The stability of different types of ST states depends on the nature of the electron/exciton-phonon (EP) coupling (e.g. deformation potential [12] against Fröhlich [13 coupling), the vibrational frequencies (e.g. acoustic vs. optic), the dimensionality $(D)$ of the lattice and other parameters. A detailed classification of ST states and ST criteria is presented, e.g., in the excellent review of Rashba [2]. In particular, from a scaling treatment of a continuum lattice model in the adiabatic limit, it has been shown that in multi-dimensional systems $(D>1)$ with only short-range non-polar EP interaction a ST carrier always forms a small polaron 14,15, 14. If there is an energy barrier that separates delocalized and spatial confined states, the free and ST states can coexist [16,17], as evidenced, e.g. for solid xenon, by the coexistence of two exciton luminescence bands (one narrow and the other broad) [3]. On the other hand, the presence of a long-range polar EP coupling ensures that at least the formation of large polaron states with moderate lattice deformation becomes possible. The picture is qualitatively changed when turning to low-dimensional systems. Recently it was shown that, unlike the continuum limit, the formation of a ST state in the 2D case within a model of local EP coupling is always accompanied by the formation of an energy barrier attributed to the lattice discreteness 18]. The $1 \mathrm{D}$ case is essentially different from the 2D and 3D ones. In 1D ST proceeds without overcoming a barrier. To be more precise, the ST state is the ground state of the infinite system at any value of the EP coupling. In the weak EP coupling regime its radius exceeds several times the lattice spacing, i.e., a large polaron is formed even under the non-polar interaction condition [2].

Although the basic concepts underlying the ST transition are long standing and the gross features of "large" and "small" polarons and excitons have been extensively studied, our understanding of the ST problem is still incomplete. In particular the physically most important crossover regime, characterized by intermediate EP coupling strengths and phonon frequencies, is difficult to handle theoretically due to the failure of the standard 
phase transition concept [19,20]. As yet, there exist no well-controlled analytical techniques to describe the transition region. Other problems, for example, concern the existence of a critical length for ST in spatially restricted 1D systems [21], the behaviour of the polaron kinetic energy [22], or the spectrum of light excitons under ST conditions [2].

With these motivations, in this paper we want to discuss the ST problem using numerical methods. The focus is on the Holstein model in one-dimension. By exact diagonalization of finite systems we analyze various ground state and spectral properties of the model. Since for the Holstein model exact results are very rare [20,23,24, and previous numerical studies have been limited either to small systems or to a particular parameter regime [25]29] this is a challenge by itself. Besides, we hope to gain more insight into the physics of small/large polarons and into the nature of the localization-delocalization transition.

The paper is organized as follows: In the next section we briefly introduce the Holstein model and outline our variational Lanczos approach that allows to study the ground state properties for all regimes of parameters on large lattices in a very efficient way. The numerical results will be presented in Sec. III. More precisely, the phase diagram of the Holstein model (A), the electron lattice correlations (B), the optical response (C) and the kinetic energy (D) will be discussed. The principal results are summarized in Sec. IV.

\section{MODEL AND METHODS}

With our focus on the self-trapping phenomenon in systems with only short-range nonpolar electron- or exciton-lattice interaction, it is appropriate to consider a model, where a single excess carrier is placed in an one-dimensional periodic array of identical molecular units, each having an internal vibrational degree of freedom. Introducing electron (exciton) $a_{i}^{[\dagger]}$ and phonon $b_{i}^{[\dagger]}$ destruction [creation] operators we can write Holsteins's molecular crystal model [12 in lattice site representation as

$$
\mathcal{H}=\hbar \omega_{0} \sum_{i}\left(b_{i}^{\dagger} b_{i}+\frac{1}{2}\right)-\sqrt{\varepsilon_{p} \hbar \omega_{0}} \sum_{i}\left(b_{i}^{\dagger}+b_{i}\right) n_{i}-t \sum_{\langle i j\rangle}\left(a_{i}^{\dagger} a_{j}+a_{j}^{\dagger} a_{i}\right)
$$


$\left(n_{i}=a_{i}^{\dagger} a_{i}\right.$; below $\left.\hbar=1\right)$. In the case of electrons the Holstein Hamiltonian (1) has been studied extensively as a paradigmatic model for small polaron formation. Here $t$ denotes the nearest-neighbour free-electron transfer amplitude, $\varepsilon_{p}$ is the strong-coupling polaron binding energy (in the atomic limit $t=0$ ), and $\omega_{0}$ is the bare phonon frequency of the dispersionsless optical phonon mode. Referring to excitons we have in mind the small-radius (Frenkel or charge transfer) excitons only, and neglect, in the lowest order of approximation, the internal structure of the exciton, i.e., we consider it as a single neutral particle.

Generally speaking, the ground-state and spectral properties of the model (1) are governed by three ratios (control parameters) defined from the bare energy scales $t, \omega_{0}$, and $\varepsilon_{p}$. First the adiabaticity parameter

$$
\alpha=\omega_{0} / t
$$

determines which of the two subsystems, excitons/electrons or phonons, is the fast and which is the slow one. In the adiabatic limit $\alpha \ll 1$, the motion of the particle is affected by quasi-static lattice deformations (adiabatic potential surface) depending on the strength of the particle-phonon interaction. On the contrary, in the anti-adiabatic limit $\alpha \gg 1$, the lattice deformation is presumed to adjust instantaneously to the position of the carrier. Conveniently the particle is referred to as a "light" or "heavy" electron/exciton in the adiabatic or non-adiabatic regimes [2]. The second parameter is the dimensionless EP coupling constant

$$
\lambda=\varepsilon_{p} / W
$$

where $W=2 D t$ denotes the half-width of the electron (exciton) band in a rigid $D_{-}$ dimensional lattice. Let us stress that $\lambda$ represents the ratio between "localization" energy $\left(\propto \varepsilon_{p}\right)$ and bare kinetic energy $(W)$ of a single particle. Both, $\lambda$ and $\alpha$, are commonly used as parameters within a perturbative analysis of the Holstein model in the limits of weak $(\lambda \ll 1)$ and strong $(\lambda \gg 1)$ EP couplings. In the latter case two different approaches, based on expansions in powers of $(\alpha \ll 1)$ and $(1 / \lambda \ll 1)$, have been elaborated for the adiabatic [12,16] and non-adiabatic [30] regimes, respectively. A third parameter, 


$$
g^{2}=\varepsilon_{p} / \omega_{0}
$$

will show to be crucial in the strong-coupling situation. $g^{2}$ determines the relative deformation of the lattice which surrounds the particle.

In the limit of small particle density, a crossover between essentially delocalized carriers and quasi-localized particles is known to occur from early quantum Monte Carlo calculations [31], provided that the two conditions $\lambda \gtrsim 1$ and $g \gtrsim 1$ are fulfilled. So, while the first condition is more restrictive if $\alpha$ is small, i.e. in the adiabatic case, the formation of a small ST state will be determined by the second criterion in the anti-adiabatic regime [29,32, 33.

It is not surprising, that the standard perturbative techniques are less able to describe the system close to the crossover region, where the energy scales are not well separated $(\lambda \sim 1, g \sim 1)$. Therefore we will apply in the following two distinct numerical methods that allow investigating the ST phenomenon on finite clusters with great accuracy.

The first method is a variational Lanczos technique (developed originally for the Holstein t-J model [28, [34]), which enables us to study the ground-state properties of fairly large systems. In recent work this technique has been adapted successfully to treat the lattice degrees of freedom in the generalized double-exchange Hamiltonian commonly used for the description of colossal magnetoresistance materials [35]. In the case of the pure Holstein model, as a first step, we perform an inhomogeneous variational Lang-Firsov (IVLF) transformation

$$
\tilde{\mathcal{H}}=\mathcal{U}^{\dagger} \mathcal{H} \mathcal{U}, \quad \mathcal{U}=\mathrm{e}^{-\mathcal{S}_{1}\left(\Delta_{i}\right)} \mathrm{e}^{-\mathcal{S}_{2}(\gamma)} \mathrm{e}^{-\mathcal{S}_{3}(\tau)}
$$

with

$$
\begin{aligned}
\mathcal{S}_{1}\left(\Delta_{i}\right) & =-\frac{1}{2 g \alpha} \sum_{i} \Delta_{i}\left(b_{i}^{\dagger}-b_{i}\right), \\
\mathcal{S}_{2}(\gamma) & =-\gamma g \sum_{i}\left(b_{i}^{\dagger}-b_{i}\right) a_{i}^{\dagger} a_{i}, \\
\mathcal{S}_{3}(\tau) & =-\ln \tau^{-1 / 2} \sum_{i}\left(b_{i}^{\dagger} b_{i}^{\dagger}-b_{i} b_{i}\right),
\end{aligned}
$$

(rescaling $\mathcal{H}=\mathcal{H} / t$ and measuring, in what follows, all energies in units of $t$ ). In a certain sense the canonical transformation $\mathcal{U}$ is a (variational) synthesis of two different approaches developed in the adiabatic [6, 36, 12, 16] and anti-adiabatic [7,8,37] theories of ST 
polarons/excitons. $\mathcal{S}_{1}$ introduces a set of static site-dependent displacement fields $\Delta_{i}$ related to local lattice distortions. This transformation ensures the correct behaviour in the adiabatic limit. That is, $\mathcal{S}_{1}$ describes the ST of "light" excitons/electrons under the conditions that (i) the electronic bandwidth significantly exceeds the characteristic phonon frequency and (ii) the lattice deformation energy is large (which allows one to treat the lattice vibrations quasi-classically). Within polaron theory such type of ST quasiparticle is often called adiabatic Holstein polaron (AHP) [12]. $\mathcal{S}_{2}$, on the other hand, describes the ST process in the anti-adiabatic limit. The variational parameter $\gamma$ (with $0 \leq \gamma \leq 1$ ) is introduced as a measure of the non-adiabatic phonon dressing of "heavy" particles, designated as "localized" excitons [38] or non-adiabatic Lang-Firsov small polarons (NLFP). For $\gamma=1$, the well-known Lang-Firsov displaced-oscillator transformation results. In addition, we have applied the two-phonon squeezing transformation $\mathcal{S}_{3}(0 \leq \tau \leq 1)$ 39,40. The squeezing phenomenon is a many particle effect being of special importance at intermediate EP coupling strengths. This effect can be seen as a phonon frequency softening and tends to offset the (polaron) band narrowing. As a second step, we approximate the eigenstates $|\tilde{\Psi}\rangle$ of $\tilde{\mathcal{H}}$ by the variational states

$$
\left|\tilde{\Psi}_{V}\right\rangle=\left|\tilde{\Psi}_{p h}\right\rangle \otimes\left|\tilde{\Psi}_{e l}\right\rangle
$$

Then, performing in a third step the average over the transformed phonon vacuum, $\overline{\mathcal{H}} \equiv$ $\left\langle\tilde{\Psi}_{p h}^{0}|\tilde{\mathcal{H}}| \tilde{\Psi}_{p h}^{0}\right\rangle$, we obtain the effective (electronic/excitonic) Hamiltonian

$$
\begin{aligned}
\overline{\mathcal{H}}= & \frac{\alpha}{4}\left(\tau^{2}+\tau^{-2}\right) N+\frac{1}{8 \lambda} \sum_{i} \Delta_{i}^{2}-(1-\gamma) \sum_{i} \Delta_{i} n_{i} \\
& -2 \lambda \gamma(2-\gamma) \sum_{i} n_{i}-\mathrm{e}^{-g^{2} \gamma^{2} \tau^{2}} \sum_{\langle i j\rangle}\left(a_{i}^{\dagger} a_{j}+a_{j}^{\dagger} a_{i}\right) .
\end{aligned}
$$

In (10), the first term leads to an increase of the zero-point energy of the phonons if $\tau^{2}<1$. The second and third contributions give the elastic energy and the particle-lattice interaction, respectively, both owing to the static lattice deformation. As a result of the incomplete LF transformation we get a constant (polaronic) level shift and an exponential band renormalization (fourth and fifth terms). Even the simplified model (10) cannot be solved 
exactly. Therefore we carry out a Lanczos diagonalization on finite $N$-site lattices using periodic boundary conditions. Employing the Hellmann-Feynman theorem, the $N+2$ variational parameters can be obtained by iteratively solving the following set of self-consistency equations:

$$
\begin{aligned}
\Delta_{i} & =4 \lambda(1-\gamma) \bar{n}_{i}, \\
\gamma & =\frac{\alpha\left[1-\bar{E}_{\Delta n} / 4 \lambda\right]}{\alpha-\tau^{2} \mathrm{e}^{-g^{2} \gamma^{2} \tau^{2}} \bar{E}_{k i n}}, \\
\tau^{2} & =\frac{\alpha}{\sqrt{\alpha^{2}-8 \lambda \gamma^{2} \mathrm{e}^{-g^{2} \gamma^{2} \tau^{2}} \bar{E}_{k i n} / N}}
\end{aligned}
$$

where

$$
\begin{aligned}
\bar{n}_{i} & =\left\langle n_{i}\right\rangle_{\overline{\mathcal{H}}} \quad \text { with } \quad \sum_{i} \bar{n}_{i}=1, \\
\bar{E}_{k i n} & =-\sum_{\langle i j\rangle}\left\langle\left(a_{i}^{\dagger} a_{j}+a_{j}^{\dagger} a_{i}\right)\right\rangle_{\overline{\mathcal{H}}}, \\
\bar{E}_{\Delta n} & =\sum_{i}\left\langle\Delta_{i} n_{i}\right\rangle_{\overline{\mathcal{H}}},
\end{aligned}
$$

denote the local particle density, the kinetic energy, and the EP interaction contribution to the ground state energy, respectively. Note that each iteration step involves the exact diagonalization of $\overline{\mathcal{H}}\left(\gamma, \tau^{2},\left\{\Delta_{i}\right\}\right)$. We note further that the Hamiltonian (10) potentially contains symmetry-broken states which originate from inhomogeneous displacement fields $\Delta_{i} \neq 0$. Therefore we have to work with an unsymmetrized set of basis states.

The second method we are going to use in the computational work is the direct numerical diagonalization of the initial Holstein Hamiltonian (1). On the one hand this should bring out valuable information on the applicability of various approximative analytical and numerical approaches. In particular we would like to test the quality of IVLF-Lanczos scheme described so far. On the other hand, combining our exact diagonalization (ED) algorithm with the Chebyshev recursion and maximum entropy methods [41], we are able to discuss dynamical properties of the systems, e.g. the optical conductivity, in more detail. Moreover, ED provides the only reliable tool for treating the transition regime. Differently from the IVLF-Lanczos treatment the translational invariance of the system is ensured. The ED 
method, based on a well-controlled truncation procedure of the phonon Hilbert space, has been described elsewhere [42,32,33]. Using parallel computers, we are able to diagonalize systems with a total dimension of $10^{10}$.

\section{NUMERICAL RESULTS AND DISCUSSION}

\section{A. Phase diagram}

In the numerical work we start with a discussion of the ground-state properties of the transformed Hamiltonian (10). Applying the IVLF-Lanczos technique presented in the previous section, we have determined the phase diagram of the 1D Holstein model. The results are depicted in Fig. 1. First let us consider the regimes II and III, corresponding to large and small polarons, respectively. Just for brevity we will use in the following the "polaron terminology", keeping in mind that all statements hold for the case of Frenkel excitons as well. The distinct types of polarons, found in II and III, may be characterized by the spatial extension and strength of the (inhomogeneous) lattice displacements $\Delta_{i}$ and by the magnitude of the polaron variational parameter $\gamma$ (see below). From exact analytical [20] and numerical [31,42,29] (cf. also Sec. III D) results it is well known that the large-size polaron turns continuously into a small-size polaron with increasing EP coupling. Since there is no true phase transition between large and small ST states at $\alpha>0$, the transition line (stars) shown in Fig. 1 only indicates the crossover region, which gets wider as the phonon frequency $(\alpha)$ increases. Within our IVLF-Lanczos treatment, the transition line has been fixed by the criterion $\Delta_{1} / \Delta_{0}=1 / e$. Performing a finite-size analysis of the II $\rightleftharpoons$ III transition, we found that the results obtained for the 64-site lattice almost agree with the extrapolated values for the infinite system. Via Eq. (11), $\Delta_{i}$ is directly related to the polaron density at site $i$. Of course, this condition can only give a crude estimate of the "transition" from large to small polarons, in particular in the non-adiabatic regime where the static $\Delta_{i}$ are less significant. According to the importance of the $\Delta_{i}(\alpha \leq 1)$ and $\gamma(\alpha \geq 1)$ effects, 
the small polaron formed in region III will be called adiabatic Holstein polaron (AHP) and non-adiabatic Lang-Firsov polarons (NLFP), respectively.

As a peculiarity of our finite system a further strongly finite-size dependent transition to a nearly free electron state (I) is observed by lowering the EP coupling strength. In other words, it seems that a critical coupling $\lambda_{c}(N)$ or equivalently a critical system size $N_{c}(\lambda)$ exists for self-trapping in 1D. Indeed, for the 1D continuum model, where the ST problem can be described by a non-linear Schrödinger equation, it has been shown recently by Rashba [21] that the ST condition is

$$
\lambda>\lambda_{c}=\pi^{2} / 2 N .
$$

This relation holds rigorously within the adiabatic theory [21] and is reproduced by our lattice model calculation as well (cf. inset Fig. 1). At $\alpha=0$, the nearly free electron phase (I) corresponds to a solution with $\gamma=0$ and $\Delta_{i}=\Delta=4 \lambda / N$. The kinetic energy, however, is unrenormalized. Our IVLF-Lanczos scheme allows to extend the above considerations to the finite phonon frequency regime. Again, at low EP couplings, we found a nearly free electron phase with a small uniform level shift $\left(\propto-2 \lambda\left[\gamma(2-\gamma)+2(1-\gamma)^{2} / N\right]\right)$. More significantly, since we have $\gamma>0$ now, the inclusion of non-adiabatic phonon effects slightly renormalizes the electron bandwidth $\bar{W}=4 \exp \left\{-g^{2} \gamma^{2} \tau^{2}\right\}$. If $\lambda$ becomes larger than $\lambda_{c}(\alpha)$ the ST proceeds by a monotonic lowering of the total energy without overcoming a ST barrier. The scaling of $\lambda_{c}(\alpha)$ with $N$ is shown in the inset of Fig. 1. In the thermodynamic limit $N \rightarrow \infty$ we obtain $\lambda_{c}(\alpha) \rightarrow 0$, i.e., in an infinite $1 \mathrm{D}$ system ST takes place at any finite value of the EP coupling.

To elucidate in more detail the different nature of polaronic states occurring in the ground-state phase diagram of the effective model (10), we present in Fig. 2 the behaviour of the variational parameters.

First of all, we should emphasize that, studying the single-electron problem, the squeezing effect $\left(\tau^{2}<1\right)$ is only of minor importance. This is obvious from (13), which yields $\tau^{2}=1$ in the thermodynamic limit. For finite systems, the leading $(1 / N)$-corrections 
$\left(\propto g^{2} \gamma^{2} \mathrm{e}^{-g^{2} \gamma^{2} \tau^{2}} \bar{E}_{k i n} / \alpha N\right)$ tends to zero in the weak- and strong-coupling adiabatic and anti-adiabatic limits.

The spatial extension of the static lattice deformation $\left(\Delta_{i}\right)$ is visualized in Figs. 2 (a) and (b) for different EP couplings corresponding to the nearly free, large and small polaron cases. As discussed above, the $\Delta_{i}$ are being constant for $\lambda<\lambda_{c}(\alpha)$ (phase I). For the large-size polaron (phase II), the lattice displacements fits extremely well to the relation

$$
\Delta_{i}=\Delta_{0} \operatorname{sech}^{2}\left[\lambda_{e f f} \cdot i\right]
$$

where $\lim _{\alpha \rightarrow 0} \lambda_{e f f}(\lambda, \alpha)=\lambda$. It is worth emphasizing, that the functional form (18), which has been derived in the framework of an adiabatic continuum theory [16, 13, 18], also describes the displacement fields in the non-adiabatic large polaron regime. Obviously, $\lambda_{\text {eff }}$ defines a characteristic inverse length scale in the system, i.e., the radius of the large polaron is approximately given by $\sim\left(2 \lambda_{e f f}\right)^{-1}$. For $\alpha=0.1$ and $\lambda=0.25$ we got $\lambda_{\text {eff }} / \lambda \simeq 0.935$. On the other hand, at $\alpha=3$ and $\lambda=2.5$, the effective coupling becomes strongly reduced: $\lambda_{e f f} / \lambda \simeq 0.116$. In the strong-coupling regime, we observe an exponential decay, $\Delta_{i} \simeq$ $\Delta_{0} \mathrm{e}^{-i / \xi}$, of the lattice distortion away from the polaron site (see insets), where $\xi$ denotes the small polaron radius. We found $\xi \simeq 0.29$ (0.19) for $\lambda=1.5$ (5.0) and $\alpha=0.1$ (3.0), i.e., in both cases the ST state is mainly confined to a single lattice site. While, in the framework of our interpolating theory, the $\Delta_{i}$ can be taken as a measure of the "adiabatic character" of the polaronic quasiparticle, its "non-adiabatic part" is described by the Lang-Firsov variational parameter $\gamma$ shown in Fig. 2 (c). Of course, in the case of "light" electrons $(\alpha<1)$, the non-adiabatic polaron effect is rather small. In particular for $\lambda>1$, when the small AHP is formed, $\gamma$ becomes strongly suppressed. Here the renormalization of the polaron band is mainly driven by the static displacement fields $\Delta_{i}$. Otherwise, for "heavy" electrons, we observe larger values of $\gamma$, which increase with increasing EP coupling strength. As a result the free electron band is transformed into a renormalized polaron band. Due to the (generalized) Franck-Condon factor $\mathrm{e}^{-g^{2} \gamma^{2} \tau^{2}}$, the bandwidth is exponentially small under strong coupling conditions $\left(g^{2}, \lambda \gg 1\right)$, where also the $K$-dependent corrections to 
the band dispersion become negligible [33]. Within our variational approach we found a first-order transition to the AHP state at extremely strong EP interaction $\left(\lambda_{I I / I I I} \simeq 4\right.$, for $\alpha=3$ ). However, it should be noted that this sharp transition is in some sense an artifact of our IVLF-scheme that compares the ground-state energies of the AHP and NLFP states, both obtained in the lowest order of approximation (remind that by deriving (10) we have performed the average over the zero-phonon state only). Including higher-order corrections, the NLFP with $\gamma \rightarrow 1$ is stabilized in the non-adiabatic strong-coupling regime (cf. the discussion in Sec. III D).

\section{B. Electron lattice correlations}

In looking for a characterization of the different polaronic regimes for the quantumphonon Holstein model (1), we have calculated the (normalized) correlation function between the electron position $i=0$ and the oscillator displacement at site $j$,

$$
\chi_{0, j}=\frac{\left\langle n_{0}\left(b_{0+j}^{\dagger}+b_{0+j}\right)\right\rangle}{2 g\left\langle n_{0}\right\rangle},
$$

by means of direct diagonalization technique. Here fermion and boson degrees of freedom

are related by the well-known relation $\left\langle b_{i}^{\dagger}+b_{i}\right\rangle_{\mathcal{H}}=2 g\left\langle n_{i}\right\rangle_{\mathcal{H}}(=2 g / N$ for the single electron case).

Alternatively, working with the effective Hamiltonian (10), the electron-lattice correlation function (19) can be expressed as

$$
\bar{\chi}_{0, j}=\gamma \delta_{0, j}+\frac{\Delta_{j}}{4 \lambda}
$$

Hence we can use the static correlation functions $\chi_{0, j}$ and $\bar{\chi}_{0, j}$ to test the accuracy of the IVLF-scheme.

Figure 3 shows the static correlation functions (19) and (20) in the adiabatic (a) and non-adiabatic (b) regimes for several coupling parameters $\lambda$ corresponding to the different polaronic "phases" indicated in Fig. 1. For parameters close to the adiabatic weak-coupling 
regime (phase I), the amplitude of $\chi_{0, j}$ is extremely small and the spatial extent of the electron-induced lattice deformation is spread over the whole lattice. In the quantumphonon model (1) the coupling gives rise to a weak dressing of the electron at any finite $\alpha$. However, the carrier is not trapped due to the zero-point quantum lattice fluctuations [44. From (20) it is clear that in the effective model (10) the on-site dynamical polaron and spatially extended static displacement field contributions are well separated. Neglecting the residual polaron-phonon interaction, the IVLF-approach describes the real situation by a (variational) superposition of both effects. In the adiabatic large polaron region II a much better description of the exact behaviour is obtained. Especially when the small AHP state evolves at $\lambda \sim 1$ the IVLF results are even in quantitative agreement with the DMRG (density matrix renormalization group) data obtained very recently by Jeckelmann and White 44] (see inset Fig. 3 (a)). At this point we would like to emphasize that performing such DMRG- and, in particular, ED-calculations requires much more memory and CPUtime resources than our extreme simple and very fast IVLF-computations. In Fig. 3, the system sizes treated within the IVLF-scheme have been restricted in order to make possible a direct comparison with the available ED/DMRG data. Obviously, for intermediate to strong EP couplings, the IVLF-results agree almost exactly with the ED and DMRG data (Fig. $3(\mathrm{~b})$ ). Here the electron-lattice correlations become very localized and finite-size effects are less important. Although the behaviour of $\chi_{0, j}$, shown in the main part of Fig. 3 , is found to be very similar for $\lambda=1.5, \alpha=3.0$ and $\lambda=4.5, \alpha=1.0$, we would like to emphasize that both parameter sets describe completely different physical situations. The distinct nature of the corresponding small polaron states becomes apparent from the variation of the static displacement fields shown in the insets. For $\lambda=4.5$ and an intermediate (or low) phonon frequency, we observe a static lattice distortion in the vicinity of the electron only. Since $\Delta_{0} / 4 \lambda \sim 1$, in (20) the second term dominates the first one and we obtain a small AHP confined to a single site. Contrary, for $\lambda=1.5$ and $\alpha=3$, we are still in the large polaron region II due to the high phonon frequency (cf. Fig. 1 and the spatial extension of the static lattice distortion shown in the left inset of Fig. 3 (b)). Nevertheless the correlations 
between the electron and the phonon remain local. But now, since the $\Delta_{j} / 4 \lambda$ are small for all $j$, the peak structure of $\chi_{0, j}$ at $j=0$ results from the first term in (20). That means it is mainly triggered by the $\gamma$-effect $(\gamma \simeq 0.69)$. This interpretation is substantiated by our ED results yielding a mean phonon number in the ground state of about 0.625 , which clearly indicates that the zero-phonon state is still the most probable one. Therefore the approximation we applied by deriving (10) and (20) is justified.

\section{Optical response}

Extremely valuable informations on the low-energy excitations in interacting electron/exciton-phonon systems can be obtained by studying their optical response. Actually, the optical absorption of small polarons is distinguished from that of large (or quasifree) polarons by the shape and the temperature dependence of the absorption bands which arise from exciting the ST carrier from or within the potential well that binds it [1]. Furthermore, as was the case with the ground state properties, the optical spectra of light and heavy electrons/excitons differ essentially as well [2]. In the most simple weak-coupling and non-adiabatic strong-coupling limits, the absorption associated with photoionization of Holstein polarons is well understood and the optical conductivity can be analyzed analytically 45 49] (for a detailed discussion of small polaron transport phenomena we refer to the review of Firsov [50]). Serious problems, caused, for example, by the complicated behaviour of the adiabatic potential surface, arise if one tries to calculate the spectrum of self-trapped light excitons [2]. Moreover, the intermediate coupling and frequency regime is as yet practically inaccessible for a rigorous analysis. On the other hand, previous numerical studies of the optical absorption in the Holstein model were limited to very small 2- or 4-site clusters [27,29]. In order to calculate the optical conductivity numerically in a wide parameter range on fairly large systems, we have implemented our computer code, which is based on a combination of Lanczos diagonalization, Chebyshev recursion and maximum entropy methods [41], on parallel machines. 
The real part of the optical conductivity, $\operatorname{Re} \sigma(\omega)=\mathcal{D} \delta(\omega)+\sigma^{r e g}(\omega)$, can be decomposed into the Drude weight $\delta$-function at $\omega=0$ and a regular part $(\omega>0)$ written in spectral representation at $T=0$ as 51

$$
\sigma^{r e g}(\omega)=\sum_{m \neq 0} \frac{\left|\left\langle\Psi_{0}\left|i \sum_{j}\left(a_{j}^{\dagger} a_{j+x}-a_{j+x}^{\dagger} a_{j}\right)\right| \Psi_{m}\right\rangle\right|^{2}}{E_{m}-E_{0}} \delta\left(\omega-E_{m}+E_{0}\right) .
$$

In $(21), \sigma^{r e g}(\omega)$ is given in units of $\pi e^{2}$ and we have omitted the $1 / N$ prefactor. For the discussion of the optical properties it is useful to consider the spectral weight function

$$
\mathcal{S}^{r e g}(\omega)=\int_{0}^{\omega} d \omega^{\prime} \sigma^{r e g}\left(\omega^{\prime}\right)
$$

as well.

Numerical results for both, $\sigma^{r e g}(\omega)$ and $\mathcal{S}^{r e g}(\omega)$, are presented in Fig. 4. We will start with the somewhat more subtle case of light electrons.

But first let us recall that, restricting ourselves to phononic states $|s\rangle_{p h}=$ $\prod_{i=0}^{N-1} \frac{1}{\sqrt{n_{i}^{s !}}}\left(b_{i}^{\dagger}\right)^{n_{i}^{s}}|0\rangle_{p h}$ with at most $M$ phonons, a $K$-symmetrized state of the Holstein model is given as $\left|\Psi_{K}\right\rangle=\sum_{m=0}^{M} \sum_{\bar{s}=1}^{\bar{S}(m)} c_{K}^{m, \bar{s}}|K ; m, \bar{s}\rangle$, where $\bar{S}(m)=(N-1+m) ! /(N-1) ! m !$ (for more details see [33]). $K$ denotes the total momentum of the coupled EP system. Then, if the EP coupling is finite, the ground state $\left|\Psi_{0}\right\rangle$ and all excited states $\left|\Psi_{m}\right\rangle$ contain components that correspond to $m$-phonon states (with $m=\sum_{i=0}^{N-1} n_{i}^{s} \leq M, n_{i}^{s} \in[0, m]$ ) in the tensorial product Hilbert space of electronic and phononic states. When the EP coupling is small $(\lambda \ll 1)$, these multi-phonon states have less spectral weight, i.e., the phonon distribution of the ground state, $\left|c_{0}^{m}\right|^{2}(M)=\sum_{\bar{s}}\left|c_{K=0}^{m, \bar{s}}\right|^{2}$, exhibits a pronounced maximum at the zero-phonon state [33]. The maximum of $\left|c_{0}^{m}\right|^{2}(M)$ is shifted to larger values of $m$ as $\lambda$ increases.

Keeping this in mind and notice further that in (21) an optical transition can take place only within the $K=0$ sector $\left(\left|\Psi_{0}\right\rangle \equiv\left|\Psi_{K=0}\right\rangle\right)$, the peak structure of $\sigma^{\text {reg }}$ shown in Fig 4 (a) may be easily understood in connection with the single particle spectrum. For low phonon frequencies $(\alpha \ll W)$, the energy to excite one phonon lies inside the bare tight-binding band $E_{K}^{(0)}=-2 t \cos K$ and we observe a flattening of the coherent band 
structure $E_{K}$ at large momenta [32,52]. Then the coherent bandwidth $\Delta E=E_{\pi}-E_{0}$ is approximately given by $\alpha\left(\ll\left[E_{K}^{(0)}-E_{0}^{(0)}\right]\right)$, i.e. by the phonon frequency, where the states at finite momenta are predominantly "phononic" states with less "electronic" spectral weight. Thus, although in principle an optical excitation can be achieved by "adding" phonons with opposite momentum to these states (in order to reach the $K=0$ sector), the overlap to the mainly "electronic" ground state is extremely small. Therefore we found, roughly speaking, the first transitions with non-negligible weight to the free electron states and its vibrational satellites (see $S^{r e g}(\omega)$ ). This is perfectly illustrated by the inset of Fig. 4 (a). Here the first and second group of peaks is approximately located at the bare tight-binding energies, $E_{K}^{(0)}(+n \cdot \alpha)$, for the allowed wave vectors of the 8-site lattice used in the numerical calculation $(K=\pi / 4, \pi / 2, \ldots)$. Of course, with increasing the lattice size the $K$ values will become dense and we will obtain the monotonous decay of the optical absorption coefficient observed for large polarons above the photoionization threshold [4]. To understand the changes in the optical absorption in the crossover region from a large--size polaron to small AHP, the main part of Fig. 4 (a) shows $\sigma^{r e g}$ at two intermediate EP coupling parameters. In this case the coherent band structure $E_{K}$ gets stronger renormalized, but, more important, the phonon distribution function in the ground state, $\left|c_{0}^{m}\right|^{2}(M)$, becomes considerably broadened. For instance, at $\alpha=0.1$, we have $\left|c_{0}^{m}\right|^{2}(M=25) \simeq 0.008(m=0)$, $0.095(m=7), 0.008(m=18)$ and $\left|c_{0}^{m}\right|^{2}(M=25) \simeq 0.0002(m=0), 0.1(m=12)$, $0.0005(m=24)$ for $\lambda=0.9$ and $\lambda=1.0$, respectively. Therefore the overlap with excited multi-phonon states is enlarged and the optical response is enhanced. The lineshape of the absorption bands reflects the phonon distribution in the ground state, where the small oscillations are due to the discreteness of the phonon frequency. As a result, the peak structure is smeared out and the wide side--bands, belonging to different electronic momenta (e.g., $K=\pi / 4$ and $K=\pi / 2)$, merge with each other.

We now turn to the heavy electron case (Fig. 4 (b)). The inset again illustrates the behaviour in the large polaron regime $(\lambda=1, \alpha=3$; cf. Fig. 1$)$, where a rather moderate band renormalization occurs $(\Delta E \sim 2.33$ due to the flattening effect [32]). But now the phonon 
frequency is large compared to the finite-size gaps between the first mainly "electronic" excitations $\left(E_{K}-E_{0}<\alpha\right.$ for $\left.K \leq \pi / 2\right)$. Therefore, in contrast to the light electron case (cf. Fig. 4 (a)), the different absorption bands (each built up by several "electronic" $K$-levels) can be classified according to the number of phonons involved in the optical transition. As can be seen from the main part of Fig. 4 (b), the absorption spectrum for a small-size polaron is quite different from that of a large polaron. According to the results of Sec. III A, a small NLFP is formed in the strong-coupling non-adiabatic limit. Here the phonons will heavily dress the electron and, concomitantly, the "electronic character" of the resulting strongly renormalized small polaron band becomes suppressed (cf. the discussion of the $K$-dependent wave-function renormalization factor $\mathcal{Z}_{K}^{(a)}=\left|\left\langle\Psi_{K}\left|a_{K}^{\dagger}\right| \Psi_{0}\right\rangle\right|^{2}$ in Ref. [33]). For our parameters $\left(\lambda=6, g^{2}=4\right)$, the maximum in phonon distribution function is located between $m=3$ and 4 . The renormalized bandwidth is small compared to all other energy scales $(\Delta E \sim 0.0782 \ll \alpha, W)$. Since the current operator connects only states having substantial overlap as far as the phononic state is concerned, multi-phonon absorptions (i.e., non-diagonal transitions [46]) become now increasingly important in the optical response. This leads to the peak structure observed for the non-adiabatic small polaron optical conductivity in Fig. 4 (b). Obviously, the different bands are being separated by multiples of the bare phonon frequency. The height of the jumps in the $\omega$-integrated conductivity is directly related to the probability of the corresponding absorption process. We found that substantial spectral weight stays in the lower energy part of the spectrum at frequencies comparable to $\varepsilon_{p}=2 \lambda\left(\simeq m_{\max } \cdot \alpha\right)$. These absorptions, resembling to some extend a large polaron's absorption, are signatures of a ST polaron with intermediate size. In the extreme strong-coupling limit the dominant absorption process results from the transfer of the ST carrier to the neighbouring site without changing the lattice distortion. That means, the optical absorption spectrum should exhibit a single-peak structure at $\omega=2 \varepsilon_{p}=4 \lambda$, which corresponds to the lowering of the electronic energy associated with the small-polaron formation [53,47]. This feature already evolves for the coupling strength considered in Fig. 4 (b) (cf. $\Delta S^{r e g}$ for the $7^{\text {th }}$ absorption band). 


\section{Kinetic energy}

Further information about the transition from large to small polarons can be obtained from the behaviour of the polaron kinetic energy $E_{\text {kin }}$. Replacing $\overline{\mathcal{H}}$ by $\mathcal{H}$, the kinetic energy can be easily obtained from static correlation function (15). On the other hand, according to the f-sum rule, $E_{k i n}$ is directly related to the $\omega$-integrated optical conductivity,

$$
-\frac{E_{k i n}}{2}=\mathcal{S}^{t o t}=\frac{\mathcal{D}}{2 \pi e^{2}}+\mathcal{S}^{r e g}
$$

Calculating, via $(21)$ and $(22), \mathcal{S}^{r e g}=\mathcal{S}^{r e g}(\infty)$ numerically, allows us to determine the Drude weight $\mathcal{D}$ as well. Sometimes one defines an effective polaronic transfer amplitude [28,22], $t_{p, \text { eff }}=E_{\text {kin }}(\lambda) / E_{k i n}(0)$, in order to characterize the polaron mobility. In our reduced units we have $t_{p, e f f} \equiv \mathcal{S}^{\text {tot }}$. From (23) it is obvious that $t_{p, e f f}$ includes both coherent and incoherent transport processes. Hence $t_{p, e f f}$ substantially differs from the exponential factor $\mathrm{e}^{-g^{2}}$, obtained in lowest order perturbation theory, and cannot be used to determine the coherent bandwidth $\Delta E$ under strong coupling conditions $\left(\lambda, g^{2} \gg 1\right)$.

The evolution of the kinetic energy $\left(\mathcal{S}^{t o t}\right)$ as a function of the EP coupling $\lambda$ is displayed in Fig. 5 (a) and (b) for the case of light and heavy electrons, respectively. In agreement with previous numerical results [31,28, 22, 44], $E_{k i n}$ clearly shows the crossover from a large polaron, characterized by a $\mathcal{S}^{\text {tot }}$ that is only weakly reduced from its non-interacting value $\left(\mathcal{S}^{\text {tot }}(\lambda=0)=1\right)$, to a less mobile small AHP/NLFP in the adiabatic/non-adiabatic strongcoupling limit.

For low phonon frequencies $(\alpha=0.1$; Fig. $5(\mathrm{a}))$, we found a rather narrow transition region. As recently pointed out by Capone et al. [29], the decrease of $\mathcal{S}^{\text {tot }}$ in the crossover regime is driven by the sharp fall of the Drude weight. By contrast the optical absorption due to inelastic scattering processes, described by the regular part of the optical conductivity, becomes strongly enhanced [33] (see the behaviour of $\mathcal{S}^{r e g}$ ). It is worth emphasizing that the IVLF-results for $\mathcal{S}^{\text {tot }}(\lambda)$ are in excellent agreement with the ED and DMRG data.

The large to small polaron transition is considerably broadened at high phonon frequencies $(\alpha=3.0$; Fig. $5(\mathrm{~b}))$. Here the IVLF-results start to deviate from the exact ones 
when $g^{2}$ gets much larger than one, thus making the lowest-order zero-phonon approximation inherent in the IVLF-scheme less justified. As mentioned already in Sec. III A, the non-analytic jump-like behaviour at $\lambda \sim 4$ is an apparent shortcoming of the variational approach which compares ground-state energies only.

Although for large enough $g$ and $\lambda$ the simple formula $\bar{W}=W \exp \left\{-g^{2}\right\}$, which should not be identified with the "Lang-Firsov approach" [22,55], works perfectly well in the determination of the coherent bandwidth $(\Delta E \simeq \bar{W})$ 27,33], the need of going beyond the lowest order of approximation to obtain reliable results for the kinetic energy has been emphasized many times [1, 33, $44,54,55$. In the non-adiabatic strong-coupling limit $\left(g^{2} \gg 1, \alpha>1\right)$, the ground-state energy obtained within second-order perturbation theory is a tiny little bit lower than the IVLF-energy and almost coincides with the ED result. Adapting the second-order strong-coupling approach presented in our previous work [33 to the 1D case (with $\gamma=1$ ), the kinetic energy is obtained, via $E_{\text {kin }}^{S C P T}=t \partial_{t}\langle\mathcal{H}\rangle$, as

$$
E_{k i n}^{S C P T}=-\frac{4}{\alpha}\left\langle\frac{1}{s}\right\rangle_{\kappa=2 g^{2}}-\mathrm{e}^{-g^{2}}\left[2+\frac{4}{\alpha}\left\langle\frac{1}{s}\right\rangle_{\kappa=g^{2}}\right]
$$

Here $\langle\ldots\rangle_{\kappa}$ means the average with respect to the Poisson distribution with parameter $\kappa$. As can be seen from Fig. 5, at large EP interactions, the strong-coupling perturbation theory (SCPT) gives a sufficiently accurate description of $\mathcal{S}^{\text {tot }}$ in both the adiabatic and non-adiabatic regimes.

\section{SUMMARY}

The main objective of this study was to re-examine in detail the self-trapping transition of electrons and excitons in one dimension (1D) within the framework of the Holstein model by the use of computational techniques. The calculations are performed by exact diagonalizations of finite systems, where the full dynamics and quantum nature of phonons was taken into account. Therefore the results are unbiased and allow to test the applicability of a numerically much more efficient variational (IVLF) Lanczos approach proposed by the 
authors. This IVLF-Lanczos technique is designed to analyze the ground-state properties of strongly correlated electron-phonon (EP) models on fairly large lattices, including static displacement field, non-adiabatic polaron and squeezing effects.

Let us summarize the main outcome of our work.

Fig. 6 illustrates the basic physics contained in the single-particle Holstein model. This model describes an continuous transition from large to small polarons with increasing EP coupling strength. Depending on the adiabaticity of the system, $\alpha=\omega_{0} / t$, the crossover regime is determined by the more stringent of the two conditions $\lambda=\varepsilon_{p} / 2 D t$ and $g^{2}=\varepsilon_{p} / \omega_{0}$. Thus starting from "light" $(\alpha<1)$ or "heavy" $(\alpha>1)$ electrons it is possible to understand the formation of small adiabatic "Holstein" (AHP) or non-adiabatic "Lang-Firsov" (NLFP) polarons as two limiting cases of a general picture.

In the infinite 1D Holstein model the self-trapped state is the ground-state for any value of the EP coupling. By contrast, in a finite 1D Holstein system a critical length or equivalently a critical EP coupling strength exists for self-trapping, rigorously at least at $\omega_{0}=0$. This may be of importance for spatially restricted systems like $\mathrm{C}_{60}$. The large-size polaron is characterized by spatially extended lattice deformations. Within our IVLF-description, the variation of the displacement fields follows the (adiabatic) formula $\Delta_{i} \propto \operatorname{sech}^{2}\left[\lambda_{e f f} \cdot i\right]$ even in the non-adiabatic regime, but with a strongly reduced inverse length scale given by an effective coupling constant $\lambda_{\text {eff }}(\lambda, \alpha) \ll \lambda$. The mean phonon number in the large polaron ground state is rather small. In 2D and 3D Holstein systems a large polaron phase does not exist.

The small polaron state is basically a multi-phonon state characterized by strong on-site electron-phonon correlations. Due to a large local static lattice distortion the AHP becomes quasi-localized on a single site. Also the NLFP is immobile because it has to drag with it a large number of phonons in its phonon cloud.

The transition from large to small polarons is accompanied by significant changes in their optical response. Especially for "light" electrons the spectral weight of the regular part of the optical conductivity is strongly enhanced at the transition. For "heavy" electrons 
the lineshape of the optical absorption spectra is highly asymmetric in the intermediateto-strong coupling regime and therefore differs considerably from the usual small polaron hopping behaviour obtained for $g^{2} \gg 1$.

As a result of self-trapping the mobility of the charge carriers is reduced. The kinetic energy indicates that the crossover region from large to small ST states is rather narrow (broad) in the adiabatic (non-adiabatic) regimes. The formation of small adiabatic Holstein polarons is accompanied by a dramatic kinetic energy loss mainly driven by a sharp drop of the Drude weight. Since both the bandwidth and the (electronic) spectral weight of the small polaron band are exponentially reduced in the extreme strong coupling case $g^{2}, \lambda \gg 1$, coherent small polaron transport becomes rapidly destroyed by thermal fluctuations.

Finally, we would like to stress that the simple IVLF-Lanczos approach provides an exceptional good description of the ground-state properties of the Holstein model, in particular for the light electron case.

\section{ACKNOWLEDGMENTS}

The authors would like to thank J. Loos and D. Ihle for many helpful discussions. We are greatly indebted to E. Jeckelmann and S. R. White for putting their DMRG data at our disposal. Special thanks go to the LRZ München, HLRZ München, and the HLR Stuttgart for the generous granting of their computer facilities. This work was performed under the auspices of Deutsche Forschungsgemeinschaft, SFB 279. 


\section{REFERENCES}

[1] Y. A. Firsov, Polarons, Izd. Nauka, (Moscow 1975).

[2] E. I. Rashba, in E. I. Rashba and M. D. Sturge, editors, Excitons, North-Holland, (Amsterdam 1982).

[3] K. S. Song and R. T. Williams, Self-Trapped Excitons, Springer-Verlag, (Berlin 1993).

[4] D. Emin, in E. K. H. Salje, A. S. Alexandrov, and W. Y. Liang, editors, Polarons and bipolarons in High- $T_{c}$ superconductors and related materials, Canmbridge University Press, (Cambridge 1995).

[5] Y. Bar-Yam, T. Egami, J. M. de Leon, and A. R. Bishop, Lattice Effects in High-T Superconductors, p. 377-421, World Scientific, (Singapore 1992).

[6] L. D. Landau, Z. Phys. 3, 664 (1933).

[7] R. Peierls, Ann. Phys. 13, 905 (1932).

[8] J. Frenkel, Phys. Zh. Sowjet. 9, 158 (1936).

[9] A. L. Shluger and A. M. Stoneham, J. Phys. Condens. Matter 5, 1993 (1993).

[10] E. K. H. Salje, A. S. Alexandrov, and W. Y. Liang, Polarons and Bipolarons in High Temperature Superconductors and Related Materials, Cambridge Univ. Press, (Cambridge 1995).

[11] A. J. Millis, P. B. Littlewood, and B. I. Shraiman, Phys. Rev. Lett. 74, 5144 (1995).

[12] T. Holstein, Ann. Phys. 8, 325 (1959).

[13] H. Fröhlich, Adv. Phys. 3, 325 (1954).

[14] Y. Toyozawa and A. Sumi, in Proc. XII Int. Conf. Physics of Semiconductors, B. G. Teubner, (Stuttgart 1974).

[15] D. Emin and T. Holstein, Phys. Rev. Lett. 36, 323 (1976).

[16] E. I. Rashba, Opt. Spektr. 2, 78 (1957); 2, 88 (1957).

[17] Y. Toyozawa, Prog. Theor. Phys. 26, 29 (1961).

[18] V. V. Kabanov and O. Y. Mashtakov, Phys. Rev. B 47, 6060 (1993).

[19] B. Gerlach and H. Löwen, Phys. Rev. B 35, 4291 (1987); 35, 4297 (1987). 
[20] H. Löwen, Phys. Rev. B 37, 8661 (1988).

[21] E. I. Rashba, Synth. Met. 64, 255 (1994).

[22] E. V. L. de Mello and J. Ranninger, Phys. Rev. B 55, 14872 (1997).

[23] J. K. Freericks and E. H. Lieb, Phys. Rev. B 51, 2812 (1995).

[24] S. Ciuchi, F. de Pasquale, and D. Feinberg, EuroPhys. Lett. 30, 151 (1995).

[25] J. Ranninger and U. Thibblin, Phys. Rev. B 45, 7730 (1992).

[26] F. Marsiglio, Phys. Lett. A 180, 280 (1993).

[27] A. S. Alexandrov, V. V. Kabanov, and D. K. Ray, Phys. Rev. B 49, 9915 (1994).

[28] H. Fehske, H. Röder, G. Wellein, and A. Mistriotis, Phys. Rev. B 51, 16582 (1995).

[29] M. Capone, W. Stephan, and M. Grilli, Phys. Rev. B 56, 4484 (1997).

[30] A. A. Gogolin, Phys. Status Solidi B 109, 95 (1982).

[31] H. D. Raedt and A. Lagendijk, Phys. Rev. Lett. 49, 1522 (1982); H. D. Raedt and A. Lagendijk, Phys. Rev. B 27, 6097 (1983); 30, 1671 (1984).

[32] G. Wellein and H. Fehske, Phys. Rev. B 56, 4513 (1997).

[33] H. Fehske, J. Loos, and G. Wellein, Z. Phys. B 104, 619 (1997).

[34] H. Fehske. Spin Dynamics, Charge Transport and Electron-Phonon Coupling Effects in Strongly Correlated Electron Systems. Habilitationsschrift, Universität Bayreuth, (1996).

[35] H. Röder, J. Zhang, and A. R. Bishop, Phys. Rev. Lett. 76, 1356 (1996); J. Zhang, A. R. Bishop, and H. Röder, Phys. Rev. B 53, R8840 (1996).

[36] S. I. Pekar, Zh. Eksp. Teor. Fiz. 16, 335 (1946).

[37] I. G. Lang and Y. A. Firsov, Zh. Eksp. Teor. Fiz. 43, 1843 (1962).

[38] A. S. Davydov, Theory of molecular excitons, McGraw-Hill, (New York 1962).

[39] H. Zheng, Solid State Commun. 65, 731 (1988).

[40] D. Feinberg, S. Ciuchi, and F. de Pasquale, Int. J. Mod. Phys. B 4, 1317 (1990).

[41] R. N. Silver et al., J. of Comp. Phys. 124, 115 (1996).

[42] G. Wellein, H. Röder, and H. Fehske, Phys. Rev. B 53, 9666 (1996).

[43] A. H. Castro Neto and A. O. Caldeira, Phys. Rev. B 46, 8858 (1992). 
[44] E. Jeckelmann and S. R. White, cond-mat/9710058 (1997).

[45] D. Emin, Phys. Rev. B 48, 13691 (1993).

[46] G. D. Mahan, Many-Particle Physics, Plenum Press, (New York, London 1990).

[47] H. G. Reik and D. Heese, J. Phys. Chem. Solids 28, 581 (1967).

[48] J. Loos, Z. Phys. B 71, 161 (1988).

[49] H. Fehske et al., Z. Phys. B 94, 91 (1994).

[50] Y. A. Firsov, Semiconductors 29, 515 (1995).

[51] E. Dagotto, Rev. Mod. Phys. 66, 763 (1994).

[52] W. Stephan, Phys. Rev. B 54, 8981 (1996).

[53] M. I. Klinger, Phys, Lett. 7, 102 (1963).

[54] A. S. Alexandrov and N. F. Mott, Polarons and Bipolarons, World Scientic, (Singapore 1995).

[55] Y. A. Firsov and E. K. Kudinov, Fiz. Tverd. Tela 39, xxx (1997). 


\section{FIGURES}

FIG. 1. Phase diagram of the 1D Holstein model. Nearly free polarons, large polarons, and small polarons exist in the regions I, II, and III, respectively. Results are obtained for finite rings with $N=32(\circ), 64(\square, *), 96(\triangle)$, and $128(\nabla)$ sites using the IVLF-Lanczos method. The inset shows the critical coupling for self-trapping, $\lambda_{c}$, at $\alpha=0(\times), 0.1(\diamond)$, $1.0(\triangleright)$, and $3.0(\triangleleft)$. The solid line gives the relation (17). For further explanation see text.

FIG. 2. Variation of the displacement fields $\Delta_{i}$ away from the central site 0 for several characteristic EP couplings $\lambda$ in the adiabatic (a) and non-adiabatic (b) regimes. The dependence of the Lang-Firsov polaron variational parameter $\gamma$ on $\lambda$ is depicted in (c).

FIG. 3. Electron-lattice correlations $\chi_{0, j}$ in the adiabatic weak-to-intermediate EP coupling (a) and non-adiabatic intermediate-to-strong EP coupling (b) cases. IVLF-Lanczos results are compared with ED data and the DMRG results taken from Ref. [44].

FIG. 4. Optical absorption in the 1D Holstein model. For $N=8$ and $M=25$, the regular part of the conductivity $\sigma^{r e g}$ (thin lines) and the integrated spectral weight $S^{r e g}$ (thick lines) are plotted as a function of $\omega$ at different EP couplings for the "light" (a) and "heavy" (b) electron cases.

FIG. 5. Kinetic energy [in units of $(-W)$ ], $S^{\text {tot }}$, and contribution of $\sigma^{\text {reg }}$ to the $\mathrm{f}-$ sum rule, $S^{r e g}$, as a function of the EP coupling, $\lambda$, in the adiabatic (a) and non-adiabatic (b) regimes. Circles (stars) denote exact (DMRG 44) data obtained for a lattice with $N=8$ (32) sites. IVLF-Lanczos results (solid curves) are compared with the predictions

of standard first--order $\left(\bar{W} / W=\exp \left\{-g^{2}\right\}\right.$; thin solid line) and second order (chain-dashed curves) perturbation theory.

FIG. 6 Schematic phase diagram of the Holstein model. 